\title{
Networks identify productive forum discussions
}

\author{
Adrienne Traxler, ${ }^{1, *}$ A. Gavrin, ${ }^{2}$ and Rebecca Lindell ${ }^{3}$ \\ ${ }^{1}$ Department of Physics, Wright State University, 3640 Colonel Glenn Hwy, Dayton, Ohio 45435, USA \\ ${ }^{2}$ Department of Physics, Indiana University Purdue University Indianapolis, \\ 402 N. Blackford St., Indianapolis, Indiana 46202, USA \\ ${ }^{3}$ Tiliadal STEM Education Solutions, Lafayette, Indiana 47901, USA
}

(Received 23 February 2018; published 10 September 2018)

\begin{abstract}
Discussion forums provide a channel for students to engage with peers and course material outside of class, accessible even to commuter and nontraditional populations. Forums can build classroom community and aid learning, but students do not always take up these tools. We use network analysis to compare three semesters of forum logs from an introductory calculus-based physics course. The networks show dense structures of collaboration that differ significantly between semesters, even though aggregate participation statistics remain steady. After characterizing network structure for each semester, we correlate students' centrality - a numeric measure of network position-with final course grade. Finally, we use a backbone extraction procedure to clean up "noise" in the network and clarify centrality-grade correlations. We find that more central network positions are positively linked with course success in the two semesters with denser forum networks. Centrality is a more reliable indicator of grade than non-network measures such as postcount. Backbone extraction destroys these correlations, suggesting that the noise is in fact signal and further analysis of the discussion transcripts is required.
\end{abstract}

DOI: 10.1103/PhysRevPhysEducRes.14.020107

\section{INTRODUCTION}

Online learning has received little attention from physics education researchers relative to topics such as conceptual understanding or student discussions in the classroom [1]. Physics courses are comparatively rare in online offerings, in part because of the hands-on laboratory courses required by the introductory sequence. However, many instructors are interested in promoting more student discussion in their classes, and web-based forums are readily available for this purpose [2]. Some work in physics has analyzed student discussion posts about homework problems [3] or in textbook annotation [4], but more general-purpose forums of the type commonly discussed in distance learning literature are only beginning to be studied $[5,6]$.

Electronic forums are included with learning management systems at universities, and are free on various standalone platforms. Thus, they are available to instructors regardless of their choice of homework system or textbook. To better understand these tools, this paper turns to network methods, which are a natural framework for analyzing the intricate record of transactions produced by discussion

\footnotetext{
*adrienne.traxler@wright.edu
}

Published by the American Physical Society under the terms of the Creative Commons Attribution 4.0 International license. Further distribution of this work must maintain attribution to the author(s) and the published article's title, journal citation, and DOI. forums [7]. We use the methods of social network analysis, a field of study that characterizes social groupings in terms of "nodes" and the "links" that connect them. We describe how we cast the forum data into these structures in a later section. Once the network is defined, we can characterize it in terms of its connectedness and the "centrality" of nodes. We will introduce several definitions of centrality, but all are efforts to measure the importance or positional advantage of a node. We also attempt to extract a network backbone, the set of links that carry the most important information about the network. One limitation of the network approach is that it does not use the content of discussions, only the structure. We also limit our analysis to the full-semester aggregated data, leaving time development of the network to future study.

We analyze forum transcripts from three semesters of an introductory calculus-based physics course. We explore and compare the structure of discussions between semesters using network analysis, drawing on the electronic "map" of student connectivity. Since online environments have not been extensively studied in physics, we will first summarize key results and questions of interest from educational technology and distance learning. This paper applies network analysis in a new context for physics educationonline learning-and shows that networks can reveal significant changes in discussion structure that are not detected by other methods. Most forum-based educational studies focus on fully online graduate courses in distance learning programs, so our work tests those predictions in an 
understudied context (a large introductory course where forum use is optional). Our data spans semesters where the hoped-for link between participation and success appeared and where it did not, allowing us to make more specific claims about which instructor behavior was associated with more productive networks. Finally, the structure of our data requires somewhat different analyses than those used for the survey-based networks typical in education studies. We discuss these differences and some measures for characterizing more complex networks. Our goal is to begin building a physics-specific understanding of how students form asynchronous discussion communities that help their learning.

\section{A. Computer-mediated communication}

Research about online student talk is typically published under keywords such as computer-mediated communication (CMC) and computer-supported collaborative learning (CSCL). Many CSCL studies compare online to offline classes in terms of student achievement or satisfaction and find that the online environment does at least as well as face-to-face classes [8]. Potential strengths of forums include longer "think time" and the ability to easily reference comments from previous weeks, while drawbacks include reluctance to participate and high variability in comment quality $[2,9]$. The reduced-social-cues nature of text communication creates an unpredictable social gestalt in CMC. Researchers have observed both impersonal, highly task-focused environments, and equally strong interpersonal groups where a sense of community can even interfere with "on-task" discussions if members hesitate to disagree with each other [10]. A review by Walther [10] synthesizes early results to suggest that the speed and quality of community development are shaped by a sense of shared purpose among users, longevity of the group, and outside cues or facilitation.

Educational discussion forums vary in formality from technical, highly focused project work to free-for-all socializing. The resulting conversation styles range from expository to epistolary [11]. Shared purpose might be expected as a given in course forums, but in practice is often missing, and this is one area where instructor guidance can be very influential [2,9]. To track the cognitive level of discussions, many researchers have turned to content analysis. Key results from this area are summarized by DeWever et al. [12] in their review of 15 content analysis frameworks for asynchronous discussion groups. They find that analysis schemes vary widely in how clearly they connect learning theory to content codes and how (or if) they report interrater reliability measures. Few schemes were used in more than one study, and there is no wide consensus about how to break online conversations into an appropriate "length scale" (post, sentence, etc.) for analysis [13].

Many researchers instead seek purely quantitative ways to study online talk, including social network analysis.
Garton et al. [7] argue that social network analysis can effectively describe online interactions with concepts like tie strength, multiplexity (different channels or purposes of communication), or structural roles of nodes in the network. Wortham [14] notes that different network topologies could support claims about communities of practice or cognitive apprenticeship. Though network analysis does not speak to the details of messages between students, it can show who talks to whom, the density and frequency of those ties, and how they evolve over time. For instructors trying to build a useful community for an online or onlinesupplemented course, there are many open questions, some of them first posed decades ago [9,15]: What timescales are appropriate to characterize discussions? What does reciprocity in relationships mean online, where many students might read a post but give no signal? How much instructor involvement is needed to promote useful conversation?

In this study, we include data from the entire semester, to eliminate possible selection effects from only sampling a slice of weeks. The question of reciprocity is taken up again in Sec. II B where our network model is discussed. We found no obvious link between the instructor's posting frequency and the discussion network that develops, but a future content analysis of the data may better address this question. A final caution in generalizing from the CSCL literature is that most results come from fully online courses, and small graduate-level courses are overrepresented. It may be possible to draw on the discussion strengths of forums without the isolating effects of a distance course by using a web-based forum to supplement a traditional live class. Studies of this type of forum use are still relatively rare $[9,16]$, especially at the introductory undergraduate level. This adjunct or "anchored" mode may be of the most interest to physics educators, whose courses are typically offered face to face and who increasingly want to build community as part of active learning.

\section{B. Network analyses of online learning}

In a recent review of social network analyses in educational technology, Sie et al. [17] classify study goals as a combination of visualization, analysis, simulation, or intervention. The work we review here fits in the first two types, and can be grouped into two broad categories: descriptive studies of network structure in online education, and research connecting students' network positions with performance measures. Studies in the first category have used network methods to probe online community structures (or lack thereof). Network analyses have shown power relations in the group and the engagement level of learners $[14,18]$. Other work contrasts between semesters or between student groups within a semester $[19,20]$, and uses visual displays or clustering analysis to show differences in the community structure. These studies show proof of concept for analyzing online talk via networks, and some suggest best practices for constructing learning 
environments, but they are primarily exploratory. They also span a range of communication channels, from synchronous text chat to asynchronous forums or email lists. One larger pattern that emerges from our review is that the communication medium affects network models. For example, using emails to link the network may produce many one-way but few reciprocal connections. We will return to this issue in Sec. II.

A second category of studies links students' network centrality with marker(s) of course success. Centrality is a numeric measure of network position, detailed in later Sec. II C. Common centrality metrics include degree (number of connections), closeness (distance to all other nodes), and betweenness (a "brokerage" position between groups of nodes). Yang and Tang [16] correlated centrality in friendship, advice, and adversarial networks with course grade in an undergraduate business course that used a forum to supplement the face-to-face class. They found that a more central position in the advice network was positively correlated with performance in both online and offline class activities. Centrality in the adversarial network (e.g., "Which of the following individuals are difficult to keep a good relationship with?') was negatively correlated with final exam and overall grade. Cho et al. [21] collected survey-based networks at the beginning and end of a two-semester online course sequence on aerospace system design. They looked for links between centrality and final grade and between a willingness-tocommunicate (WTC) construct and network growth. They found that postcourse (but not precourse) degree and closeness centrality were positively correlated with final grade, and that students with higher WTC were more likely to form new ties during the two semesters.

Other approaches use different positive outcomes or look for network characteristics of successful students rather than course-wide correlations. Dawson [22] correlated students' centrality in course forum networks and their sense of course community as measured by Rovai's Classroom Community Scale [23]. He found that degree and closeness centrality were positively correlated and betweenness centrality was negatively correlated with greater feelings of classroom community. However, the data pools 25 courses at undergraduate and graduate levels, different amounts of online integration, and different communication channels, so direct comparisons with these results are difficult. In a second study [24], the same author examined student participation in an optional (but encouraged) discussion forum used as a supplement to a large introductory chemistry course. Focusing on the "ego networks" (immediate connections, see Ref. [25]) of individual students in the top and bottom $10 \%$ of the grade distribution, he found that students in the high-performing group had larger ego networks, and the members of those networks had higher average grades. Additionally, there was a higher percentage of instructor presence in the networks of high-scoring students, who tended to ask a larger number of conceptual questions. Students in the lower-performing group often asked more fact-based questions which were typically answered by other students, leading to an unintended "rich get richer" effect of the higher-performing students receiving a larger share of instructor attention.

There is evidence of networks' ability to distinguish between at least some types of online dialog structure, and to support links between network position and final grade. The latter point has been observed in some physics classrooms [26], but not previously sought in electronic forums. With some exceptions [20], most of the online network studies either give results for a single course offering or pool multiple courses together. They provide interesting cases, but it is unclear how stable their results may be from one semester to the next. Since network analysis requires start-up time for data cleaning and analysis, it is also reasonable to ask if it shows anything new compared to the participation statistics reported by most forum software. Building on the literature above, we consider three research questions:

(1) How do discussion forum networks differ among multiple semesters of an introductory physics course, and can this information be extracted more easily from participation statistics?

(2) How much are student final grades correlated with their centrality in the discussion forum network?

(3) Do centrality-grade correlations, if present, strengthen when reducing the network to a more simplified "backbone?"

The third question has not been considered in any prior educational network studies we could find, but emerged from the high density of our discussion networks (Sec. III) and recent work piloting network sparsification in physics education research [27].

\section{METHODS}

Here, we describe how we collected data, built forum networks, calculated and used network measures, and simplified the network using backbone extraction. Further details on the backbone process, including source code, are in the Supplemental Material [28].

\section{A. Data collection}

We adopted the CourseNetworking (CN) platform [29], which combines a robust forum tool with features typical of learning management systems. $\mathrm{CN}$ is a cloud-based platform, accessible either through a web browser or through apps on IOS and Android mobile devices. We selected CN primarily because the interface is "student centric," that is, student work occupies the majority of the view, and faculty focused tools are secondary. Although it is possible to use $\mathrm{CN}$ as a standalone LMS, the instructor coupled it with another system (Canvas) and used CN 
exclusively as a forum. The $\mathrm{CN}$ forum has a look and feel similar to other popular social media, so students pick it up with minimal introduction. The forum supports starting threads as either posts or polls and allows hyperlinks, embedded images and videos, and downloadable files. Polls may be structured as multiple choice, ranking, free response, and other formats, allowing students to create and post "sample questions" for one another. Students may also post reflections (comments) to threads and rate them using a 1-3 star system.

One of us (A. G.) used the $\mathrm{CN}$ forum in three sections of a calculus-based introductory mechanics class. The initial enrollment was over 160 students each semester, mostly engineering and computer science majors. Each semester, approximately $85 \%$ of the students who enrolled completed the course. The institutional context is an urban, public university enrolling approximately 30000 students. In all three semesters, the university had undergraduate racial or ethnic demographics of $71 \%-72 \%$ white, $10 \%$ African American, 6\%-7\% Hispanic or Latino, other groups (including international students) $4 \%$ or less. The majority of students commute, and most work part- or full-time in off campus jobs [30].

The course was heavily interactive, using Peer Instruction [31] and Just-in-Time Teaching (JiTT) [32] in the lectures and group problem solving in the recitations. The course used WebAssign [33] for homework, with the text by Tipler and Mosca [34] linked as an e-book through WebAssign. Hour exams and the final were traditional paper-and-pencil tests. The exams were closed book, with a standard "formula sheet" provided. There were 28 problem sets assigned each semester, due Sunday and Thursday nights. Labs met weekly for two hours and were graded on individually completed worksheets and reports. Recitation sections were based on informal group work. Problems were projected on screen, and students worked in selfassembled groups of 3-4 on whiteboards mounted around the room. Each problem concluded with 2-3 multiple choice questions. Students answered these using the same clicker technology used for Peer Instruction in the lecture. Typical classes included two such problems.

The LMS provided by the university was Canvas. This was used primarily for its gradebook function, and for a few "high stakes" messages during the semester. $\mathrm{CN}$ was used for all day-to-day course communications, including making announcements, distributing handouts, etc. Grading was based on exams (30\%), a comprehensive final (20\%), problem sets (15\%), labs (15\%), JiTT warmup exercises (10\%), clicker questions (5\%), and recitation scores (5\%). Students earned extra credit proportional to their participation in the forum up to a maximum of 5\%; additional participation was encouraged but no further incentives were offered. All calculations involving student grades first remove any forum bonus points.
Information about $\mathrm{CN}$ was provided in the syllabus and during the first lecture. Students were told that CN would be the favored communications tool and informed of the $5 \%$ extra credit incentive. The first lecture included a brief (approximately 3 minute) demonstration of $\mathrm{CN}$, but no technical instructions were provided. In Semesters 1 and 3, the instructor used the CN "Tasks" feature to suggest an optional weekly discussion topic, which took place in the forum and did not involve extra class time. Finally, in Semester 3, the first-day introduction included mention of a new ability in the software to tag posts with instructor- or user-created "hashtags." In all other respects, the CN implementation was identical across terms.

\section{B. Casting forum data as networks}

The forum transcript contains the following data: Content ID (post, poll, or reflection); a unique student identifier code; the date, time, and text of the post; the number of attachments; and the star rating (pre-2016, number of "likes") of the post or comment. The analysis in this paper uses the content ID, student code, date, and time. The transcript groups all reflections below their parent post or poll, showing a threaded view that corresponds to the student view of the forum. $\mathrm{CN}$ logs track the "nesting" level of a reply (whether a student hit the reply button for the original post, or for another reply to that post). In practice, most students did not organize their replies in a multilayer fashion, using a single reply layer even when the content was clearly a response to another comment. Because of this behavior, we treat each thread as consisting of a root plus single reply level (Fig. 1, left). This shapes network structure-by contrast, some studies with clear nested structure in the electronic transcripts have drawn links only between a poster and the person to whom they were immediately replying [[18,20], Fig. 1, right]. In our data, accurate nesting information is largely unavailable, requiring an alternate model for drawing connections between participants in a thread.

Though it is intuitive that students talking in a forum are interacting with each other somehow, some set of assumptions must be chosen to map the logs into a network object.

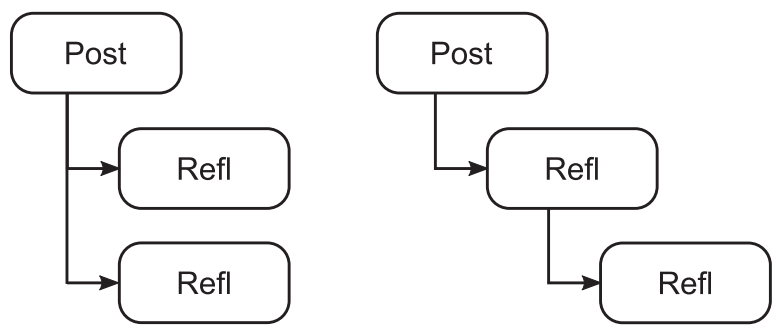

FIG. 1. Structure of forum transcript data. The $\mathrm{CN}$ data largely shows a post with a single reply layer (left), in contrast to studies where more nested structure is retained and informs the network construction (right). 

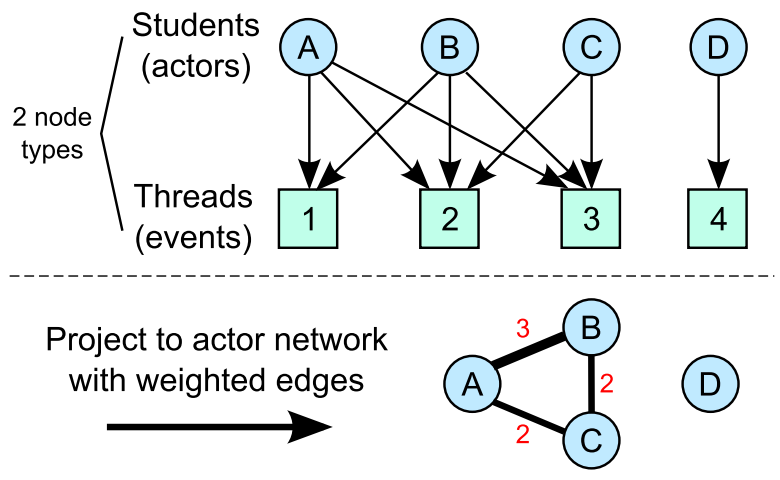

FIG. 2. Bipartite network model for transforming forum transcript into a network object. Students are "actor" nodes, who post to thread or "event" nodes. The actor network projection links together student nodes who posted to the same thread.

Prior studies of forum networks have used several different approaches: adding a link between a student commenter and the poster they were directly replying to $[18,20]$, surveying students at the beginning and end of the semester $[16,21]$, or unspecified methods [22,35]. We used a bipartite network model, often used to model situations where both people ("actors") and some set of shared activities ("events") are of interest [36]. This approach has been used to model scientific collaboration networks, where scientists and papers are the node types. More recently, some online education studies have used it to capture student forum interactions [37,38].

The bipartite analysis proceeds in two stages, as shown in Fig. 2. First, the network is created with two types of nodes (students and threads). Next, a one-mode projection is created by linking all actors who were connected to the same event node. A student who posts multiple times in a thread will have higher-weight links to the other thread participants in the actor projection. Weights from multiple links between two people (from posting in multiple common threads) are summed. The bipartite approach gives us a heavily interlinked, noisy picture of forum conversations. From this point, centrality and other calculations continue on the actor network. This social network has more links, and more gradations of link weight, than networks drawn from recall-based survey data. The next step is deciding how to characterize the whole discussion web and the position of nodes within it.

\section{Network measures}

Even a small network quickly becomes unwieldy to describe by naming all actors and listing their connections. (But for very small classes this kind of description can be very illuminating, see Ref. [18].) Structural measures condense broad features of network objects, and centrality measures quantify the position and importance of a particular node. For a general overview of social network concepts, see Refs. [25] or [39]. We first discuss three metrics for structure to compare the different forum networks (average degree, density, and transitivity), then three node centrality measures (PageRank, Target Entropy, and Hide) that we will link to students' class performance.

\section{Whole-network measures}

The most basic descriptors of a network are the number of nodes $(N)$ and edges $\left(N_{E}\right)$. Beyond these traits, there are many possible summary measures, and which is most useful depends on the information the researcher wishes to highlight [40]. Some measures are only intended to compare networks of strictly identical size, while others relax that assumption. To evaluate the connectedness of the three forum semesters, we report average degree, density, and transitivity (also called the clustering coefficient).

Average degree is simply the number of edges divided by the number of nodes,

$$
k_{\mathrm{av}}=N_{E} / N
$$

It gives a sense of how many conversation partners a typical student may have. Though it is commonly reported for networks, it can be misleading on its own, as degree distributions in social networks tend to be highly skewed [41].

Network density is defined as the ratio of total to possible edges:

$$
\rho=\frac{N_{E}}{N(N-1) / 2}
$$

for an undirected network. Larger social networks tend to be less dense-mathematically, because the denominator of Eq. (2) scales as $N^{2}$, and practically because any individual can only sustain relationships with so many other people [42]. In a forum environment, where both rare and frequent interactions are recorded, higher density values may be expected unless some thresholding process is used (see Sec. II D).

Transitivity or clustering coefficient compares the number of closed triangles of nodes to the number of all connected node triplets $[40,43]$. It quantifies the probability that two of a student's forum "neighbors" are also linked directly to each other. Average degree tells us about how many connections a person is likely to have, but those connections could be anything from dense cliques to a "star" structure where many people communicate with a focal actor but not with each other. Transitivity goes further to tell us something about structure in the network as a whole. It provides an alternate way to think about connectedness, and unlike the more commonly reported density value, it is not sensitive to small changes in network size.

Uncertainty in all of these measures can be estimated using bootstrap methods [44]. Using this technique, a new sample of $N$ nodes is drawn from the observed network and 
an artificial network is constructed using the connections belonging to those nodes. The average degree (or density, or transitivity) of this artificial network represents a new possible value, and the process is repeated many times, generating a distribution of simulated values. This distribution can be used to calculate a standard error for the observed statistic. We use the bootstrap method of Snijders and Borgatti [44] with 5000 samples to calculate standard errors and $t$ tests to compare average degree, density, and transitivity between the three semester networks.

\section{Node-level measures}

Centrality describes the position or importance of a node in a network. The number and strength of a node's connections to others, and whether they are at the core or periphery of the whole network, form the basis of centrality. The most basic measure is degree centrality, which counts the number of edges (connections) attached to a node. In weighted networks, this concept is often expanded to node strength [25], which is the sum of all the edge weights connected to the node. In directed networks such as the reduced backbones described later, directionality of links can be tracked using in- and out-degree or inand out-strength. All of these values account for only the direct neighbors of a node, but the wider set of neighbors' connections can also constrain or boost a node's access to information or resources (for example, study group invitations). A later generation of centrality measures accounts for both the number of neighbors of a node and the importance of each of those neighbors. Their importance, in turn, depends on that of their own neighbors, requiring simultaneous solution over the whole network. Measures of this type can be computed as eigenvalue problems [45].

As with whole-network descriptors, centrality measures must be chosen from many possible options to best fit the context. Many of the online learning papers reviewed used some combination of degree, closeness, or betweenness centrality, but typically did so on unweighted networks measured at one or two time points $[16,21,22]$. There are several proposed methods to generalize these measures to weighted networks [46], but for our full-semester aggregated networks, methods that incorporate the whole network structure become more attractive [20,26]. Because of the structure of our data, and for easier comparison with prior results in physics education, we will examine PageRank, Target Entropy, and Hide centrality as described next.

PageRank is one of the most popular measures of the eigenvalue type, built on the same base algorithm used by the Google search engine to rank the importance of pages on the internet [47]. PageRank designates a node as being important if a large number of important nodes point to it. It was developed for directed networks (on the internet, linking to another page makes a directed network edge), but can be used in undirected networks as well.
Target Entropy (TE) and Hide [48], together with PageRank, have been used in network analyses of classroom interactions between physics students over a university term [26]. Target Entropy is a measure of the diversity of a node's information sources; high TE nodes will have many neighbors who themselves talk to a wide array of other students. Conversely, Hide quantifies how difficult it is to "find" a node in the network. High-Hide nodes will have few neighbors, who may themselves be more sparsely connected than average.

For each semester, we calculate PageRank, Target Entropy, and Hide for all nodes. The PageRank computation uses the igraph package in R $[49,50]$ and the other two measures use code from Ref. [26], Supplemental Material. We then calculate Pearson correlations between each centrality measure and final course grade. Network centralities inherently violate the assumption of independence that underlies standard correlation calculations. To correct for this issue, permutation tests can be used, where the data set is repeatedly resampled and the correlation recalculated, typically thousands or tens of thousands of times [51]. The resulting distribution of correlation coefficients gives an estimate of how likely the observed correlation was to occur by chance in a network of the same size and density - in other words, an empirical $p$ value. Though network measures are our primary interest, for research question 1 we also report Pearson correlations between final grade and a student's total contributions to the forum (their combined number of Posts, Polls, and Reflections).

\section{Backbone extraction}

The forum networks generated by the process described are much more dense than typical survey-based networks in a physics class of comparable size [52-54]. Since they are built from thousands of posts, with content ranging from physics-based conversations to "postcount" boosting, it seems reasonable that not all interactions are equally important. The most active individuals might be connected by some core structure underlying the "noisy" full network, and it is these types of structures that backbone extraction is designed to uncover [55].

Various methods exist for extracting backbones, and for this work we used the locally adaptive network sparsification (LANS) algorithm of Foti et al. [56], which has been tested on several real-world dense networks including answer distributions from the Force Concept Inventory [27]. LANS is tuned through a parameter $\alpha$ : for each node in the network, all edges below the $1-\alpha$ percentile of edge weight are discarded. Thus, an alpha value of 0.05 would correspond to keeping only the 95th percentile and above of a node's strongest links. For a node with edges of weights 1 , 5 , and 10, a threshold of $\alpha=0.05$ would remove all but the weight-10 edge(s). There is no single value of alpha which will suit for all network problems; rather, each 
TABLE I. Forum participation and network statistics by semester. Participation includes students enrolled $\left(N_{\text {class }}\right)$, percent who posted in the forum, total number of threads and replies, and average replies per thread and posts per student plus or minus standard deviation. Network statistics include number of nodes $(N)$, isolates, average degree $k_{\text {av }}$, network density, and transitivity. For the last three measures, numbers in parentheses indicate the standard error in the last digit.

\begin{tabular}{lccccccccccc}
\hline \hline Semester & $N_{\text {class }}$ & Part. (\%) & Threads & Replies & Replies/thread & Posts/student & $N$ & Isolates & $k_{\text {av }}$ & Density & Transitivity \\
\hline 1 & 173 & 90 & 936 & 2376 & $2.5 \pm 3.6$ & $21 \pm 16$ & 156 & 12 & $49(5)$ & $0.32(3)$ & $0.64(3)$ \\
2 & 152 & 86 & 912 & 2253 & $2.5 \pm 2.4$ & $23 \pm 24$ & 131 & 5 & $28(4)$ & $0.22(3)$ & $0.53(3)$ \\
3 & 166 & 87 & 762 & 2508 & $3.3 \pm 3.3$ & $22 \pm 22$ & 145 & 6 & $40(5)$ & $0.28(3)$ & $0.63(3)$ \\
\hline \hline
\end{tabular}

analysis should test several values and select one that simplifies to the desired density while still preserving necessary information. We test several values of $\alpha$ and repeat the permutation correlation tests between centrality and final grade, investigating whether backbone extraction strengthens the correlations by removing the effect of extraneous low-weight connections.

\section{RESULTS}

\section{A. Comparing forum participation and networks}

Table I shows summary participation statistics for the forum. Each semester, $85 \%-90 \%$ of the enrolled students posted at least once. The number of threads was similar between the first two semesters and lower in the third, when the average number of replies per thread increased. We compared thread length and posts per student between semesters using pairwise Wilcoxan tests, which account for the non-normal distribution and presence of outliers in the data. Only semester 3 had a significantly different $\left(p<10^{-5}\right)$ average number of replies per thread. There were no significant differences in the number of posts per student between semesters.

Average posts per student can mask very different posting patterns, if some semesters have a few high-volume participants and others have a lower but more widespread posting rate. Figure 3 shows the distribution of forum contributions among students. To control for varying class size, the figure shows the density, essentially a smoothed histogram normalized to integrate to 1 for each semester. All three semesters have a peak at low activity (0-15 contributions), a few very active members around 75-100 contributions, and a high-activity "tail." Semester 1 has its largest peak around 25 contributions, while the other two semesters had a less prominent "shoulder" there.

Table I also shows descriptive statistics for the forum discussion networks. Nodes are all students who posted at least once, and isolates are students who only posted one thread, which received no replies (see student D in Fig. 2). Using bootstrapped standard errors as described in Sec. II C 1, we tested for statistically significant differences in the three measures of network connectivity (average degree, density, and transitivity). Semesters 1 and 2 are significantly different on all metrics, and Semesters 1 and 3 are different on none. Semester 3 is significantly higher than 2 on average degree and transitivity, and marginally higher on density. (See Supplemental Material [28] for additional detail about these calculations.) Because larger networks will tend to have lower density, the "natural" ranking of density values in the three semesters would be $(2,3,1)$ for a comparable level of network structure. The observed ranking reverses this.

The aggregate forum network for the whole semester is too dense to be visually useful without extensive filtering of low weight edges [[5], Fig. 1]. Figure 4 shows the week 7-8 subset of semesters 1 and 2, a time of similar activity in the middle of the semester. Each circle shows a student, sized by total contributions over the semester and colored by final grade. Darker connecting lines indicate higher-weight edges, resulting from more common threads between a pair of students. Though total forum activity was similar between the two semesters, the semester 2 network is less dense and more dominated by a few high-participation, high-grade students during the time shown. Semester 3 (not

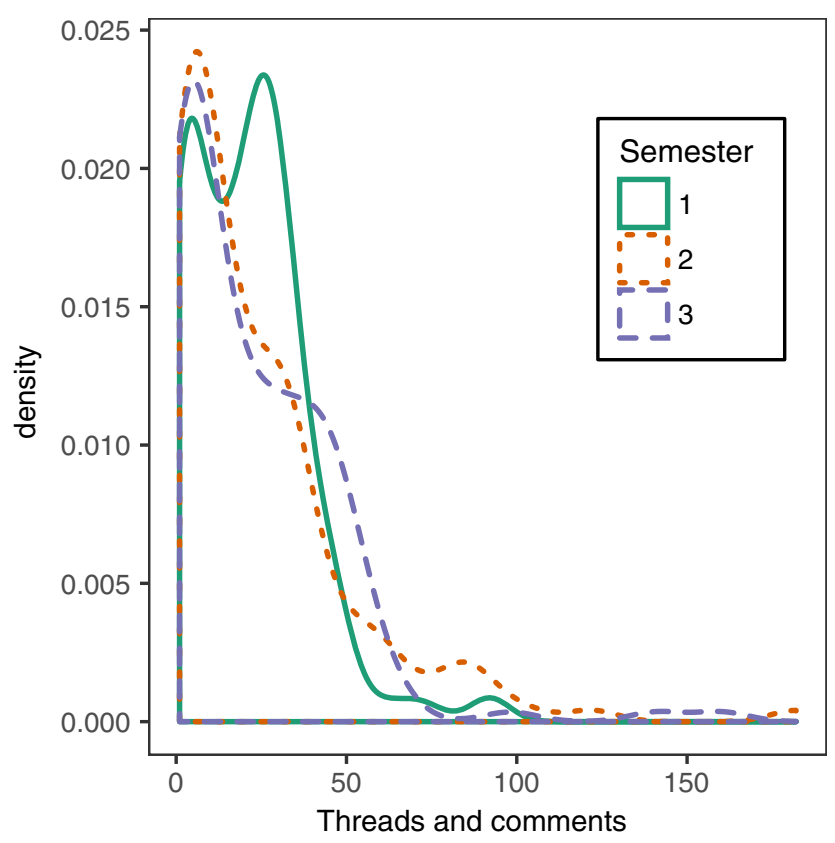

FIG. 3. Density distribution of forum activity (combined threads and comments) for class members by semester. The instructor's contribution totals are included and are 94, 182, and 141 by semester. 


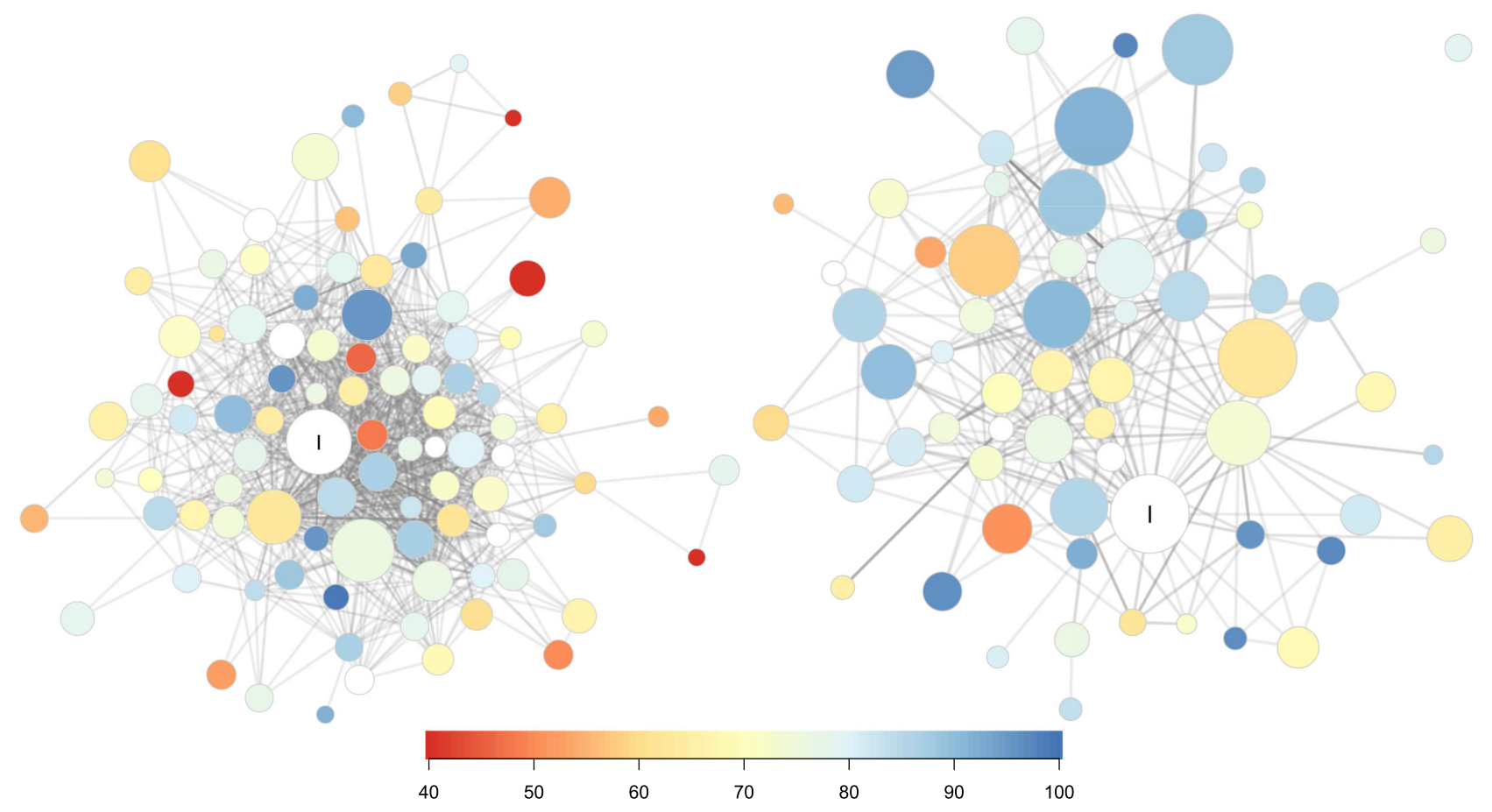

FIG. 4. Forum networks from weeks $7-8$ in semester 1 (left) and semester 2 (right). Line opacity is scaled by edge weight, so darker lines indicate more threads in common for a student pair. Nodes are sized by total contributions over the semester and colored by grade (red low, yellow medium, blue high). Nodes without grades (withdrawals and instructor or CN staff accounts) are white, and the instructor's node is labeled "I."

shown) is visually "between" the two pictures, with fewer students posting than semester 1 during this time frame but with visibly higher density than semester 2 .

\section{B. Centrality-grade correlations}

Table II shows the bootstrap correlation coefficients $r$ between final grade and centrality in the discussion forum networks. In the first semester, PageRank and Target Entropy are positively correlated with final grade and Hide is negatively correlated, all at small effect sizes. For Pearson correlations, the magnitude of the coefficient is also the effect size, and we use Cohen's suggested thresholds of $(0.1,0.3,0.5)$ for size of effect [57]. In the second semester, no correlations are significant. The third semester repeats the pattern of semester 1, with the PageRank and Hide correlations now above the threshold for medium

TABLE II. Correlation coefficients $r$ between final grade, the network centrality measures PageRank (PR), Target Entropy (TE), and Hide (H), and forum participation (total threads + comments). Asterisks show the level of statistical significance $\left({ }^{*} p<0.05,{ }^{* *} p<0.01\right.$, and $\left.{ }^{* * *} p<0.001\right)$.

\begin{tabular}{lllll}
\hline \hline Semester & \multicolumn{1}{c}{$r_{\mathrm{PR}}$} & \multicolumn{1}{c}{$r_{\mathrm{TE}}$} & \multicolumn{1}{c}{$r_{\mathrm{H}}$} & \multicolumn{1}{c}{$r_{\mathrm{Part}}$} \\
\hline 1 & $0.18^{*}$ & $0.29 * *$ & $-0.27 * *$ & 0.091 \\
2 & 0.13 & 0.17 & -0.18 & 0.12 \\
3 & $0.34^{* * *}$ & $0.28^{* *}$ & $-0.31^{* *}$ & $0.33^{* * *}$ \\
\hline \hline
\end{tabular}

effect size. The table also gives the Pearson correlation between total number of forum contributions and final grade for each semester. This correlation is only significant in semester 3, at a medium effect size.

\section{Backbone extraction}

The goal of backbone extraction is to simplify a network to its essential structure, so high-density forum networks are ideal candidates for this technique. For each semester of data, we calculated the LANS backbone extraction at values of $\alpha=(0.5,0.1,0.05,0.01)$. Table III shows the number of edges and the fraction of the original total edge weight remaining [55] for each reduction of the three semesters.

There are competing criteria for judging a backbone extraction to be appropriate or a value of alpha to be suitably small. One heuristic is that a large portion of the original network weight (the sum of its weighted degree) should remain [55]. Another possible metric is to lower $\alpha$ until the forum network reaches a comparable density or average degree to a classroom survey-based network of similar size [53]. By the first measure, values of $\alpha=0.05$ or lower may be cause for concern in this data, since they hold only one-quarter of the original network weight (a small amount in comparison to the example backbones of Serrano et al. [55]). By the second measure, values of $\alpha=0.05$ or 0.01 might be most appropriate. 
TABLE III. Edges $\left(N_{E}\right)$ and fraction of total original weight $\left(\% W_{T}\right)$ at each level of backbone extraction; $\alpha=1$ is the original network.

\begin{tabular}{lrrrrrrrr}
\hline \hline & \multicolumn{2}{c}{ Semester 1 } & & \multicolumn{2}{c}{ Semester 2 } & & \multicolumn{2}{c}{ Semester 3 } \\
\cline { 2 - 3 }$\alpha$ & $N_{E}$ & $\% W_{T}$ & & $N_{E}$ & $\% W_{T}$ & & $N_{E}$ & $\% W_{T}$ \\
\hline 1 & 7628 & 1.00 & & 3704 & 1.00 & & 5858 & 1.00 \\
0.5 & 5635 & 0.88 & & 2476 & 0.88 & & 4042 & 0.88 \\
0.1 & 1173 & 0.36 & & 572 & 0.39 & & 1000 & 0.39 \\
0.05 & 661 & 0.24 & & 334 & 0.26 & & 530 & 0.25 \\
0.01 & 194 & 0.09 & & 186 & 0.12 & & 221 & 0.10 \\
\hline \hline
\end{tabular}

To resolve this possible contradiction, the ultimate arbiter is what happens to the centrality values of the nodes: their relative distribution and their correlations with students' final grades. For all three semesters, backbone reduction appears to destroy rather than strengthen correlations between network centrality and final grade. The negative Hide or grade correlation vanishes immediately, with similar though less severe effects on PageRank and Target Entropy (see Supplemental Material [28] for details). In the third semester, there is some suggestion that backbone reduction does not hurt and may even help the PageRank and Target Entropy correlations down to $\alpha=0.1$. However, the overall effect of the technique is to reduce rather than highlight the useful information.

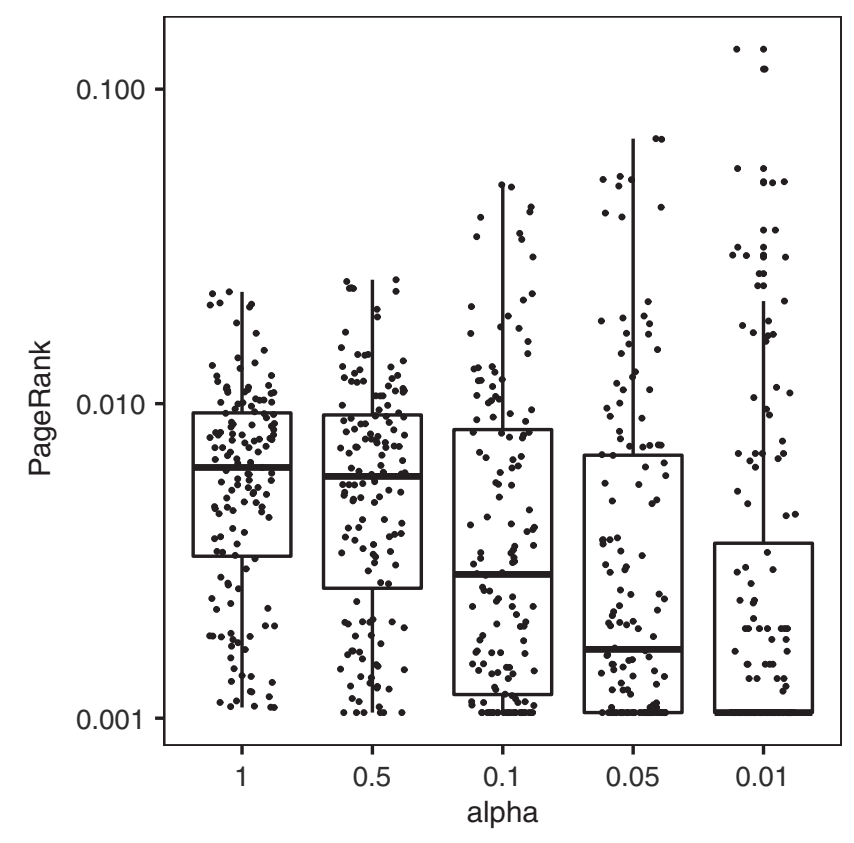

FIG. 5. Box plots of PageRank centrality for the semester 1 network backbones. The bottom, middle, and top line of the boxes show first quartile, median, and third quartile. The upper and lower "whiskers" extend to the maximum and minimum values or 1.5 times the interquartile range, whichever is larger. As alpha decreases, more node centralities cluster at the minimum value.
Figure 5 shows box plots of the PageRank scores of nodes for the original $(\alpha=1)$ and reduced networks for semester 1. These distributions help to explain why correlations with final grade decrease as supposedly "extraneous" links are removed. Backbone extraction flattens calculated centrality values for most nodes in the network as $\alpha$ decreases, with the distribution skewing lower and many nodes eventually occupying the minimum possible PageRank value. Plots for the other semesters and the other two centrality measures show a similar effect.

\section{DISCUSSION}

\section{A. Network analysis reveals important differences in forum use between semesters}

Our first research question was, How do discussion forum networks differ among multiple semesters of an introductory physics course, and can this information be extracted more easily from participation statistics? From the analyses summarized in Table I and Fig. 4, we find that the forum networks have different connectedness and breadth of participation between semesters. In particular, semesters 1 and 3 show a higher level of connectivity (by average degree, density, and transitivity) that is not easily explained by fluctuations in class size or numbers of discussion threads and comments. In contrast, non-network participation statistics show few significant differences between the classes, with only semester 3 having longer discussion threads (but not more activity overall). Some essential structure of the discussions in our data was captured by network analysis but missed by participation tracking.

Our second research question was, How much are student final grades correlated with their centrality in the discussion forum network? Students who are more central in the forum network tend to score higher in the course, but not in every semester-in particular, the higherdensity networks are those in which centrality is correlated with grade. Target Entropy and Hide seem to be the most reliable predictors, with PageRank somewhat less consistent. Exploratory analysis shows that in this data set, Target Entropy and Hide are highly correlated, so we focus our discussion here on Target Entropy. This result builds on the findings of question 1: networks better capture the discussion connectivity, but beyond that, they track a kind of interaction that benefits students in this course.

Our third research question was, Do centrality-grade correlations, if present, strengthen when reducing the network to a more simplified backbone? We predicted that using backbone extraction on the forum networks would clarify correlations between centrality and final grade, by streamlining low-weight links that proliferate in long "chat" threads and leaving the most important connections between students. We found that instead, this "noise" is part of the signal, and that reducing the forum networks to 
backbone representations destroys correlations between centrality and grade. It is possible that a backbone extraction method developed specifically for bipartite networks [58] might improve this result. However, plotting PageRank, Target Entropy, and Hide at successive alpha levels shows that backbone extraction flattens these centrality distributions and pushes more and more nodes to the minimum value. This issue seems likely to recur even with a change in algorithm.

\section{B. Implications for network research}

One recommendation that emerges from the literature review of this paper is for researchers to carefully document their choices in using network models to describe online learning. Some past studies have used survey methods to gather network data [16,21], while others draw from electronic logs $[14,18-20,24]$. Studies in the first category base their approach on earlier social network analysis studies of business organizations, though physics education researchers have tied similar data collection to theoretical frameworks of transformation of participation or communities of practice $[59,60]$.

Studies that derive their data from electronic logs are more common in the CSCL literature, and this is a promising direction given the growing amount of data that is available from learning management systems. Kortemeyer [3] argues that these data open a more natural window onto students' thought processes than think-aloud interviews, where students may be trying to perform to the interviewer's expectations. For instructors, detecting differences in student participation early in the semester, based on their use of resources like forums, can give early warnings about at-risk students in live or online courses [19,24].

A few studies do not specify how they constructed their networks. Both the data source (survey or logs) and the assumptions made about how to connect the network have consequences for the density and structures that result. In other words, the network model—what constitutes a link between students-is an interaction model [61], which makes a statement about what communication processes the researcher thinks are important to learning in a given environment. Our bipartite model generated far denser networks than survey-based classroom studies, even those drawn from weekly sampling (see Ref. [26], Supplemental Material Fig. 5 for link weight distribution of their densest network). We chose an expansive definition of interaction, and find that centrality in the resulting network is an equally strong predictor of grades as a sparser survey approach. Our measured correlations between network centrality and grades are also comparable to those found between annotation quality and exam grade in a physics content analysis study [4]. Different online learning studies have used a variety of centrality measures, and it is not at all clear that a "best" set will emerge. Only by documenting their assumptions can researchers allow for any hope of comparing between or replicating results.

\section{Implications for online learning research}

As outlined, the range of data sources, network statistics, and outcome measures makes it challenging to check results between CSCL network analyses. However, we can look for alignment in trends or effect sizes of results. Dawson [24] found that high-performing students had more connections and were more likely to be linked to the instructor. High Target Entropy students in our semesters 1 and 3, who were more likely to do well in the course, would tend to have a large number of connections like the highscoring students in Dawson's study. Similarly, low Target Entropy - signaling limited sources of information-would generally correspond to student ego networks with only a few connections.

Though the instructor in our data was not intentionally making an anchored forum with the traits recommended by Guzdial and Turns [9], the CN interface builds in two of those authors' recommendations: a thread-grouped view with always-visible archives and the ability to choose a post category (through instructor- or user-created "hashtags"). The authors make a third recommendation of "anchor" threads that prompt students with a few key discussion topics and include a link to post their contributions. In semesters 1 and 3, the instructor created anchor threads via the Tasks feature on $\mathrm{CN}$. Tasks show at the top of the forum page, and were updated once a week in those two semesters. The instructor did not use these weekly tasks in semester 2, and this change came with (though we cannot say it was the sole cause of) a loss of network connectivity.

Aviv et al. [20] compared two semesters and found that the level of integration between the forum and class assignments was linked to substantial differences in the amount and cognitive level of discussion by students. Our results match theirs in part: the raw amount of discussion was not necessarily tied to facilitation, but the resulting network between students was more dense and appears to be more educationally useful in the more-structured semesters. The work by Aviv and collaborators is one of a small but growing number of studies that combine network measures with content analysis of posts $[11,15,18]$. Work in physics has shown links between the cognitive level of student comments on homework problems [3] or textbook annotation [4] with their grades [3,4] or conceptual gains [4]. Content analysis of the $\mathrm{CN}$ data, currently in progress, will let us look for interplay between the quantitative network structures and qualitative content of discussions.

Cho et al. [21] and Yang and Tang [16] found that degree centrality positively correlated with final grade in surveybased classroom networks, though in the first study, the correlation was only marginally significant and a significant correlation instead appeared with closeness centrality. Though their network construction methods were different, 
the correlations found ( $r=0.442$ for [21], $r=0.4$ or 0.46 for [16]) are similar to the results of this study as well as the correlations with PageRank, Target Entropy, and Hide found by Bruun and Brewe [26].

The closest comparison study in physics is Bruun and Brewe [26], who used weekly surveys to build an aggregate network for an introductory mechanics course. We found that the three centrality measures that emerge as most important in their study-PageRank, Target Entropy, and Hide-are also useful for exploring position or grade correlations in the forum data. Of these, Target Entropy and Hide seem to show the most consistent signal; these measures originate from a theoretical perspective of quantifying information flow $[26,48]$, which may be especially suited for describing long post chains in forum networks.

\section{LIMITATIONS AND FUTURE WORK}

Like most CSCL studies [8], this is not a control-group experimental study. One possible reading of our results is that more engaged students tend to participate in the forum, and that high-centrality nodes are merely the "good" students (however a reader might define that) who would succeed regardless of a forum or discussion prompts. Certainly, there is evidence that students who are inclined to talk to others are more likely to benefit from forums [21]. However, the lack of centrality-grade correlations in semester 2 suggests that this explanation is incomplete. First, and as a general argument for forum use, even students who are predisposed to talk about class material can benefit from tools for doing so outside of class hours at commuter schools. Second, the differences in semester 2 show that even a similarly active forum may not be equally useful. There is no reason to believe that the fraction of engaged, self-starting students was substantially different between our three semesters, but there are significant differences in network structure and in correlations between forum position and grade. Taken together, these points suggest that not only does instructor facilitation matter, but that network analysis can detect this difference even when participation tracking does not.

The network statistics and centrality correlations in this work use the aggregated full-semester forum data. Though an important starting place, this obscures details of time development through the semester. A subsequent longitudinal analysis might look for a timescale over which the network stabilizes [62,63], whether growth follows a particular theoretical model [41], or what student clusters emerge over time [62].

Finally, analyzing the text of posts is beyond the scope of this paper, but spot-checking suggests that the most active threads (which contribute to higher network connectivity) are a mixture of physics-based and social topics. This further weakens the idea that the correlations we found only show the best students using the forum for strictly studious purposes. The nature of the conversations and community that arise are more complicated than an on- or off-topic dichotomy [64]. The next stage of this project will use posttext to analyze the discussion differences between semesters and the effect of anchoring by the instructor's weekly Tasks. Ultimately, content analysis results can be combined with a time-developing picture of the network characteristics [37] to better understand instructor facilitation, the student discussion culture that emerges, and the benefits that both have for learning in physics forums.

\section{ACKNOWLEDGMENTS}

Dr. Ali Jafari and Alice Zhao of the Course Networking development team were essential to data collection for this project.
[1] J. L. Docktor and J. P. Mestre, Synthesis of disciplinebased education research in physics, Phys. Rev. ST Phys. Educ. Res. 10, 020119 (2014).

[2] J. R. Howard, Discussion in the College Classroom: Getting Your Students Engaged and Participating in Person and Online (Jossey-Bass, San Francisco, CA, 2015).

[3] G. Kortemeyer, An analysis of asynchronous online homework discussions in introductory physics courses, Am. J. Phys. 74, 526 (2006).

[4] K. Miller, S. Zyto, D. Karger, J. Yoo, and E. Mazur, Analysis of student engagement in an online annotation system in the context of a flipped introductory physics class, Phys. Rev. Phys. Educ. Res. 12, 020143 (2016).

[5] A. Traxler, A. Gavrin, and R. Lindell, CourseNetworking and community: Linking online discussion networks and course success, in Proceedings of the Physics Education Research Conference 2016, Sacramento, CA, edited by D. L. Jones, L. Ding, and A. Traxler, pp. 352-355, DOI: 10.1119/perc.2016.pr.083.

[6] A. Gavrin and R.S. Lindell, Connecting students' homework to their participation in a course-based social network, in 2017 ASEE Annual Conference \& Exposition (Columbus, OH, 2017).

[7] L. Garton, C. Haythornthwaite, and B. Wellman, Studying online social networks, J. Computer-Mediated Commun. 3 (1997).

[8] G. M. Johnson, Synchronous and asynchronous text-based CMC in educational contexts: A review of recent research, TechTrends : for leaders in education and training 50, 46 (2006). 
[9] M. Guzdial and J. Turns, Effective discussion through a computer-mediated anchored forum, J. Learn. Sci. 9, 437 (2000).

[10] J. B. Walther, Computer-mediated communication: Impersonal, interpersonal, and hyperpersonal interaction, Communication research 23, 3 (1996).

[11] P. J. Fahy, Epistolary and expository interaction patterns in a computer conference transcript, Int. J. E-Learning Distance Educ. 17, 20 (2002); http://ijede.ca/index.php/ jde/article/view/181/118.

[12] B. De Wever, T. Schellens, M. Valcke, and H. Van Keer, Content analysis schemes to analyze transcripts of online asynchronous discussion groups: A review, Comput. Educ. 46, 6 (2006).

[13] L. Rourke, T. Anderson, D. R. Garrison, and W. Archer, Methodological issues in the content analysis of computer conference transcripts, Int. J. Artificial Intelligence Educ. 12, 8 (2001).

[14] D. W. Wortham, Nodal and matrix analyses of communication patterns in small groups, in Proceedings of the 1999 Conference on Computer Support for Collaborative Learning, CSCL '99 (International Society of the Learning Sciences, Palo Alto, California, 1999); https://telearn .archives-ouvertes.fr/hal-00197319.

[15] R. E. Rice and G. Love, Electronic emotion: Socioemotional content in a computer-mediated communication network, Communication research 14, 85 (1987).

[16] H.-L. Yang and J.-H. Tang, Effects of social network on students' performance: A web-based forum study in Taiwan, J. Asynchronous Learn. Networks 7, 93 (2003); https://onlinelearningconsortium.org/read/journal-issues/.

[17] R. L. L. Sie, T. D. Ullmann, K. Rajagopal, K. Cela, M. Bitter-Rijpkema, and P. B. Sloep, Social network analysis for technology-enhanced learning: Review and future directions, Int. J. Technol. Enhanced Learning 4, 172 (2012).

[18] M. de Laat, V. Lally, L. Lipponen, and R.-J. Simons, Investigating patterns of interaction in networked learning and computer-supported collaborative learning: A role for Social Network Analysis, Int. J. Computer-Supported Collab. Learn. 2, 87 (2007).

[19] C. Reffay and T. Chanier, Social network analysis used for modelling collaboration in distance learning groups, in Proceedings of the 6th International Conference on Intelligent Tutoring Systems, ITS '02 (Springer-Verlag, London, 2002), pp. 31-40.

[20] R. Aviv, Z. Erlich, G. Ravid, and A. Geva, Network analysis of knowledge construction in asynchronous learning networks, J. Asynchronous Learn. Networks 7, 1 (2003).

[21] H. Cho, G. Gay, B. Davidson, and A. Ingraffea, Social networks, communication styles, and learning performance in a CSCL community, Comput. Educ. 49, 309 (2007).

[22] S. Dawson, A study of the relationship between student social networks and sense of community, Educ. Technol. Soc. 11, 224 (2008); http://www.jstor.org/stable/pdf/ jeductechsoci.11.3.224.pdf.

[23] A. P. Rovai, Development of an instrument to measure classroom community, Internet Higher Educ. 5, 197 (2002).
[24] S. Dawson, "Seeing" the learning community: An exploration of the development of a resource for monitoring online student networking, Br. J. Educ. Technol. 41, 736 (2010).

[25] R. A. Hanneman and M. Riddle, Introduction to Social Network Methods (University of California, Riverside, Riverside, CA, 2005), http://faculty.ucr.edu/ hanneman/.

[26] J. Bruun and E. Brewe, Talking and learning physics: Predicting future grades from network measures and Force Concept Inventory pretest scores, Phys. Rev. ST Phys. Educ. Res. 9, 020109 (2013).

[27] E. Brewe, J. Bruun, and I. G. Bearden, Using module analysis for multiple choice responses: A new method applied to Force Concept Inventory data, Phys. Rev. Phys. Educ. Res. 12, 020131 (2016).

[28] See Supplemental Material at http://link.aps.org/ supplemental/10.1103/PhysRevPhysEducRes.14.020107 for details on comparing networks, centrality structure of backbone networks, and backbone extraction source code.

[29] Coursenetworking, Http://www.thecn.com.

[30] A. W. Radford, M. Cominole, and P. Skomsvold, Demographic and Enrollment Characteristics of Nontraditional Undergraduates: 2011-12, Technical Report No. NCES 2015025 (National Center for Education Statistics, Washington, DC, 2015).

[31] E. Mazur, Peer Instruction: A User's Manual (Prentice Hall, Upper Saddle River, NJ, 1997).

[32] G. Novak, A. Gavrin, W. Christian, and E. Patterson, JustIn-Time Teaching: Blending Active Learning with Web Technology, 1st ed. (Addison-Wesley, Upper Saddle River, NJ, 1999).

[33] Webassign, Http://www.webassign.net.

[34] P. A. Tipler and G. Mosca, Physics for Scientists and Engineers, 6th ed. (W. H. Freeman, New York, 2008).

[35] P. J. Fahy, G. Crawford, and M. Ally, Patterns of interaction in a computer conference transcript, Int. Rev. Res. Open and Distributed Learn. 2, 1 (2001).

[36] S. P. Borgatti and M. G. Everett, Network analysis of 2-mode data, Soc. Networks 19, 243 (1997).

[37] F. Ouyang and C. Scharber, The influences of an experienced instructor's discussion design and facilitation on an online learning community development: A social network analysis study, Internet Higher Educ. 35, 34 (2017).

[38] D. Rodríguez, M. Á. Sicilia, S. Sánchez-Alonso, L. Lezcano, and E. García-Barriocanal, Exploring affiliation network models as a collaborative filtering mechanism in e-learning, Interact. Learn. Environ. 19, 317 (2011).

[39] S. Wasserman and K. Faust, Social Network Analysis: Methods and Applications, Structural Analysis in the Social Sciences (Cambridge University Press, New York, NY, 1994).

[40] L. da F. Costa, F. A. Rodrigues, G. Travieso, and P. R. Villas Boas, Characterization of complex networks: A survey of measurements, Adv. Phys. 56, 167 (2007).

[41] M. E. J. Newman, The structure and function of complex networks, SIAM Rev. 45, 167 (2003).

[42] R. I. M. Dunbar, Neocortex size as a constraint on group size in primates, J. Hum. Evol. 22, 469 (1992). 
[43] M. E. J. Newman, Scientific collaboration networks. I. Network construction and fundamental results, Phys. Rev. E 64, 016131 (2001).

[44] T. A. B. Snijders and S. P. Borgatti, Non-parametric standard errors and tests for network statistics, Connections 22, 61 (1999); http://www.insna.org/PDF/Connections/v22/ 1999_I-2_61-70.pdf.

[45] P. Bonacich, Power and centrality: A family of measures, Am. J. Sociology 92, 1170 (1987).

[46] T. Opsahl, F. Agneessens, and J. Skvoretz, Node centrality in weighted networks: Generalizing degree and shortest paths, Soc. Networks 32, 245 (2010).

[47] S. Brin and L. Page, The anatomy of a large-scale hypertextual Web search engine, in Seventh International WorldWide Web Conference (WWW 1998) (Brisbane, Australia, 1998).

[48] K. Sneppen, A. Trusina, and M. Rosvall, Hide-and-seek on complex networks, Europhys. Lett. 69, 853 (2005).

[49] R Core Team, $R$ : A Language and Environment for Statistical Computing (R Foundation for Statistical Computing, Vienna, Austria, 2017).

[50] G. Csardi and T. Nepusz, The igraph software package for complex network research, InterJournal Complex Systems, 1695 (2006).

[51] D. Z. Grunspan, B. L. Wiggins, and S. M. Goodreau, Understanding classrooms through social network analysis: A primer for social network analysis in education research, CBE Life Sci. Educ. 13, 167 (2014).

[52] E. Brewe, L. H. Kramer, and G. E. O'Brien, Changing participation through formation of student learning communities, AIP Conf. Proc. 1289, 85 (2010).

[53] A. Traxler, Community structure in introductory physics course networks, in Proceedings of the Physics Education Research Conference 2015, College Park, MD, edited by A. D. Churukian, D. L. Jones, and L. Ding (AIP, New York, 2015), pp. 331-334, DOI: 10.1119/perc.2015.pr.078.
[54] E. Sandt and A. Traxler, Non-traditional students' conceptual scores and network centrality in SCALE-UP classrooms, in Proceedings of the Physics Education Research Conference 2016, Sacramento, CA, edited by D. L. Jones, L. Ding, and A. Traxler (AIP, New York, 2016), pp. 296-299, DOI: 10.1119/perc.2016.pr.069.

[55] M. Á. Serrano, M. Boguñá, and A. Vespignani, Extracting the multiscale backbone of complex weighted networks, Proc. Natl. Acad. Sci. U.S.A. 106, 6483 (2009).

[56] N. J. Foti, J. M. Hughes, and D. N. Rockmore, Nonparametric sparsification of complex multiscale networks, PLoS One 6, e16431 (2011).

[57] J. Cohen, A power primer, Psychol. Bull. 112, 155 (1992).

[58] Z. Neal, The backbone of bipartite projections: Inferring relationships from co-authorship, co-sponsorship, co-attendance and other co-behaviors, Soc. Networks 39, 84 (2014).

[59] E. Brewe, L. Kramer, and V. Sawtelle, Investigating student communities with network analysis of interactions in a physics learning center, Phys. Rev. ST Phys. Educ. Res. 8, 010101 (2012).

[60] J. Bruun, Networks in Physics Education Research: A Theoretical, Methodological, and Didactical Explorative Study, Ph.D. thesis, University of Copenhagen, Copenhagen, Denmark, 2012.

[61] L. C. Freeman, Centrality in social networks conceptual clarification, Soc. Networks 1, 215 (1978).

[62] J. Bruun and I. G. Bearden, Time development in the early history of social networks: Link stabilization, group dynamics, and segregation, PLoS One 9, e112775 (2014).

[63] J.P. Zwolak, M. Zwolak, and E. Brewe, Educational commitment and social networking: The power of informal networks, Phys. Rev. Phys. Educ. Res. 14, 010131 (2018).

[64] L. Rourke, T. Anderson, D. R. Garrison, and W. Archer, Assessing social presence in asynchronous text-based computer conferencing, Int. J. E-Learning Distance Educ. 14, 50 (1999). 